\title{
The role of subprocess-connector in business process modeling
}

\author{
K. Shoilekova ${ }^{1}$, K. Grigorova ${ }^{1}$, E. Malysheva ${ }^{2}$ \\ ${ }^{1}$ Angel Kanchev University of Ruse, 8 Studentska str., Ruse 7017, Bulgaria \\ ${ }^{2}$ Volga Region State University of Services, 4 Gagarina str., 445677, Togliatti, Russia
}

\begin{abstract}
The paper emphases the importance of the correct and consistent business process planning. The most important objective in business process generation is the proper execution of the activities in a business organization and studying the links between them. To visualize all business processes in a system a subprocess - connector has been created that helps to detect the deadlock markings in a system. It is impossible to change a component without interfering the operation with the others. Several aspects of Petri Nets as a tool for process simulation and surmounting the deadlocks in a system are presented.
\end{abstract}

Keywords: Business Process; Petri nets; Simulation; Connector; Subprocess

\section{Introduction}

Each enterprise is based on models representing its inside processes. A company success is determined by the rational organization of its business processes that are subject to in-depth analyses and continual optimization, which is within the priorities of the business processes management but not a single-time initiative. From an enterprise point of view the management of business processes is becoming increasingly important: business process, or process for short, controls which piece of work will be performed by whom and which resources are exploited for this work, i.e. a business process describes how an enterprise will achieve its business goals.

A business process can be defined as a sequence of activities distinctly specified within an organization involving people, equipment, applications, information and other resources, aiming to create products, respectively, values.

Figure 1 shows a three-dimensional view of a business process including:

- $\quad$ case dimension;

- $\quad$ process dimension;

- resource dimension.

The case dimension signifies that all cases are handled individually. From business process point of view, cases do not interact directly. They influence each other indirectly by sharing resources and data. The process dimension specifies the activities in the workflow process, i.e. the tasks and routing along the tasks. The resource dimension signifies the resources, their roles as well as the organizational units. A business process can be visualized by a number of nodes in a three-dimensional view as shown in Fig. 1. Each node represents either a work item (case + task) or an activity (case + task + resource). It can be seen from Fig. 1 that the business process management is an adhesive of cases, tasks, and organization [1].

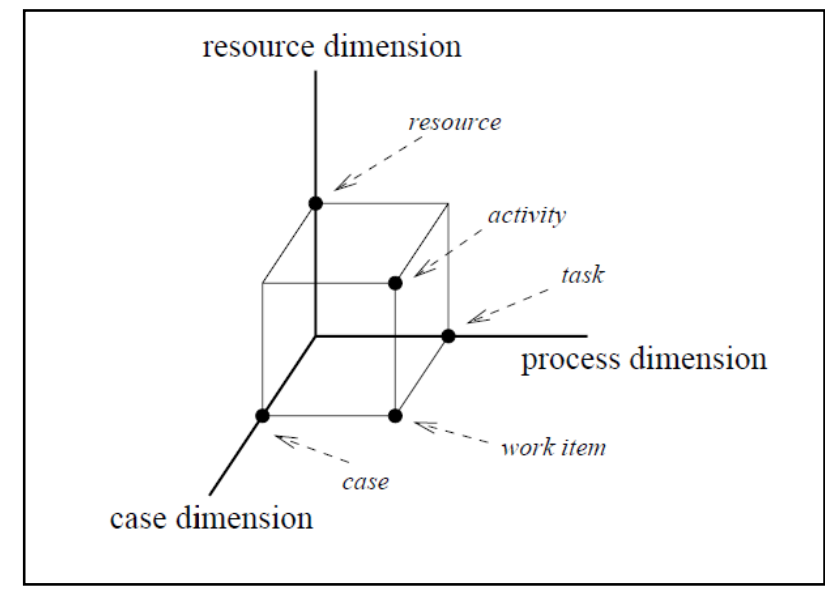

Fig. 1. A three-dimensional view of a business process [1].

\section{Petri nets}

Petri nets were introduced by Carl Adam Petri in 1962]. They became the first standard adopted for business process modeling. Since then Petri nets have been used to model and analyze different processes with applications ranging from embedded systems to flexible manufacturing systems, user interaction, and business processes. Petri nets offer a graphical representation of stepwise processes including choice, iteration and concurrent execution. They are distinguished with an exact 
mathematical definition of their execution semantics, and a well-developed mathematical theory for process analysis. Petri nets are recognized as one of the known techniques for describing business processes in a formal and abstract way [5], [6], [8], [9].

Over the last three decades the classical Petri net has been extended with color, time and hierarchy specifications [2], [4]. These extensions facilitate complex process modeling, where data and time are important factors. Petri nets are used as a powerful tool in business process modeling for several reasons. They offer:

- formal semantics;

- graphical language;

- $\quad$ support of basic primitives needed to model business processes;

- $\quad$ analysis based on four general approaches:

○ Reachability Analysis: involves the enumeration of all reachable markings, but it suffers from the state-space explosion issue;

- Matrix Equation Approach: in many cases it is applicable only to special sub-classes of Petri nets or special situations;

- Invariant Analysis: determines sets of places or transitions with special features, as token conservation or cyclical behavior;

- Simulation: discrete-event simulation is an option to check system's properties.

While modeling refers to the development of mathematical representation of modeled object processes, simulation concerns data processing by means of algorithms and procedures for solving all mathematical calculations related to the model, i.e. computer simulation is a system's representation by its model activation.

Business processes' simulation based on Petri nets is a way to show the system characteristics. The main idea is to use an appropriate execution algorithm in order to detect its unwanted properties.

Studies in [3] and [7] helped to compile a list of over 200 different techniques and tools for business processes simulation using Petri nets. Regretfully, a significant number of websites are out of operation being without support and updates for more than 15 years, or they are just source-codes lacking any descriptions, which fences off the understanding of their functions.

In result of the initial filtering, the list was reduced to 47 techniques appropriate for further examination and comparative analysis. Even these are too many for the purpose of detailed investigation, and speaking about an effective software product, at least it is expected to have gained minimum popularity within society and scientific communities. As there is no information about a worked out comparison, a number of Google Scholar results and Google search results were used.

The above stated approach reduced the filtering criteria to:

1. The tool was created or updated over the last 16 years (after year 2000).

2. There is an operational website of the tool that can be used in reality, and it is provided with relevant documentation, help menus or other scientific papers.

3. The tool has minimum 200 citations in scientific papers (ascertained with Google Scholar search).

4. The tool has minimum 5000 results ascertained with Google search.

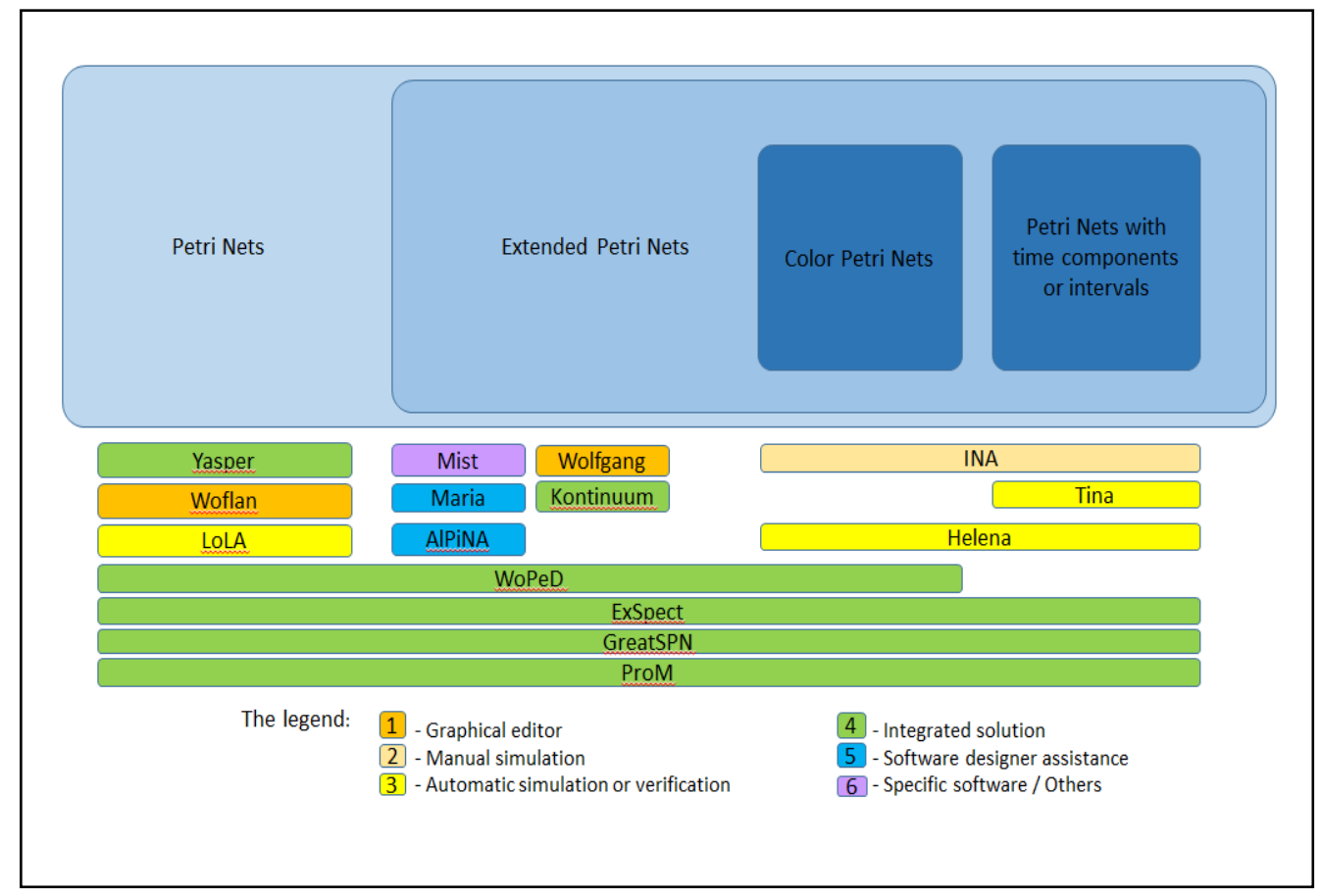

Fig. 2. Tools for business process analyses and transformations.

Concluding filtering, the final list includes 15 tools ranked by the number of Google search results:

1. Kontinuum - this tool is business process management software for use by business decision makers and administrators as a tool to maximize control, save time and cut unnecessary costs. 
Data Science / K. Shoilekova, K. Grigorova, E. Malysheva

2. Maria - Modular Reachability Analyzer is a reachability analyzer for concurrent systems that uses Algebraic System Nets (a high-level variant of Petri nets) as its modelling formalism.

3. Wolfgang - it is a lightweight tool that allows users to easily create and edit Petri nets and check them against general and workflow specific net properties.

4. Mist - it is tool to check safety properties against Petri Net like models.

5. INA - Integrated Net Analyzer is a tool package supporting the analysis of Place/Transition Nets (Petri Nets) and Coloured Petri nets.

6. Yasper - Yet Another Smart Process EditoR is a tool for modeling, analyzing and simulating automated business processes. Yasper's models are based on Petri nets.

7. ProM - Process Mining Framework is an open source framework directed to RapidMiner 5, a system supporting the design and documentation of the whole data mining process.

8. LoLA - a Low Level Petri Net Analyzer has been implemented for the validation of reduction techniques for place/transition net reachability graphs. LoLA features symmetric as well as stubborn set based methods. Net symmetries are automatically computed. Stubborn sets are customized to the particular analysis task.

9. Tina - TIme Petri Net Analyzer is a toolbox for the editing and analysis of Petri Nets, with possibly inhibitor and read arcs, Time Petri Nets, with possibly priorities and stopwatches, and an extension of Time Petri Nets with data handling called Time Transition Systems.

10. Helena - a High LEvel Net Analyzer is a model checker developed at the CNAM university in Paris. It is a free software available under the terms of the GNU general public license.

11. WoPeD - Workflow Petri Net Designer is an open source software product developed at the Cooperative State University Karlsruhe, Germany (distributed under GNU license).

12. GreatSPN - GRaphical Editor and Analyzer for Timed and Stochastic Petri Nets is a tool package for modeling, validation and performance evaluation of distributed systems.

13. ExSpect - it is a software tool designed for discrete process modeling.

14. Woflan - Workflow Analyzer is an analysis tool which can be used to verify the correctness of a workflow procedure.

15. AlPiNA - Algebraic Petri Nets Analyzer is a model checker for Algebraic Petri Nets created by the SMV Group at the University of Geneva.

After multiple filtering and exploring different tools for business processes simulation based on Petri nets, a comparative analysis was worked out (Fig. 2), and they were placed in categories: graphical editor, tools for manual simulation, tools for automatic simulation or verification, integrated solution combining previous solutions, as well as tools for software designer assistance and tools for specific software development.

The analysis confirms that Petri nets can be regarded as an appropriate tool for business processes modeling and simulation.

A main task in business process modeling is the verification of process models regarding syntactical and structural errors. A syntactical error is given if modeling elements are used in an invalid manner. While the former might be checked with low efforts, the latter usually requires a very complicated analysis to prove properties like deadlock in the models. A deadlock in a process model is given if a certain instance of the model (one or more but not necessarily all) can not continue working, while it has not yet reached its end.

Verification is concerned with determining, in advance, whether a process model exhibits certain desirable behaviours. By performing this verification at design time, it is possible to identify all or part of potential problems. In this case the model can be modified before it is used for execution. As part of the systems rely on process models for execution of work, careful analysis of process models at design time can greatly improve the reliability of such systems.

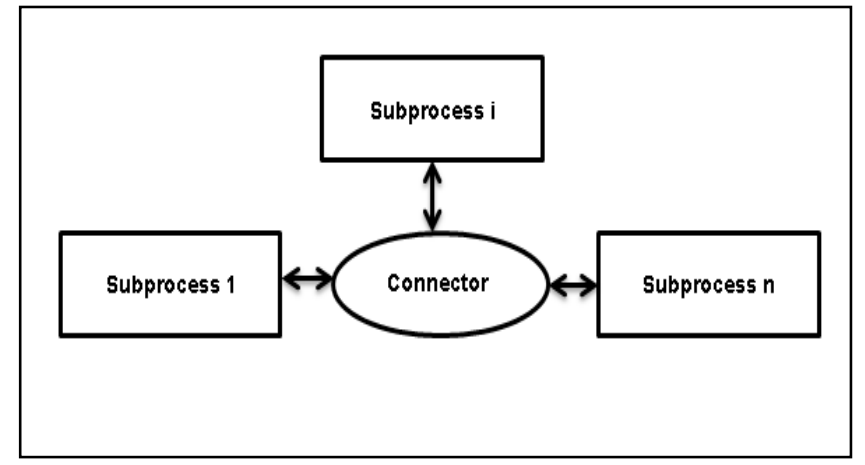

Fig. 3. Subprocess - connector.

The only drawback is that every business process is part of another process, which makes the visualization of all processes incorporating the main business process a difficult task. This problem can be solved by creating a subprocess and connector aiming to describe how business processes interact in order to achieve a complete system functionality (Fig. 3).

To manage the size and complexity of business process models, the use of subprocesses is widely advocated. But there is no solid evidence for benefits of modularization of business process models as well as clear criteria for identifying subprocesses. The modularization may foster the understanding of a complex business process model by its "information hiding" quality and 
force the effectiveness of business process modeling. It is even possible to specify some criteria that can be used to automatically derive process fragments that seem suitable to capture as subprocesses.

A subprocess - connector is composed of one basic module called connector and a number of secondary modules called subprocesses. The main business process is described within the connector while subprocesses serve to describe those business processes, which interact with the main process. The subprocess - connector is designed to show the communication between separate processes that is accomplished by determining the input and output characteristics of every other subprocess (Fig. 4).

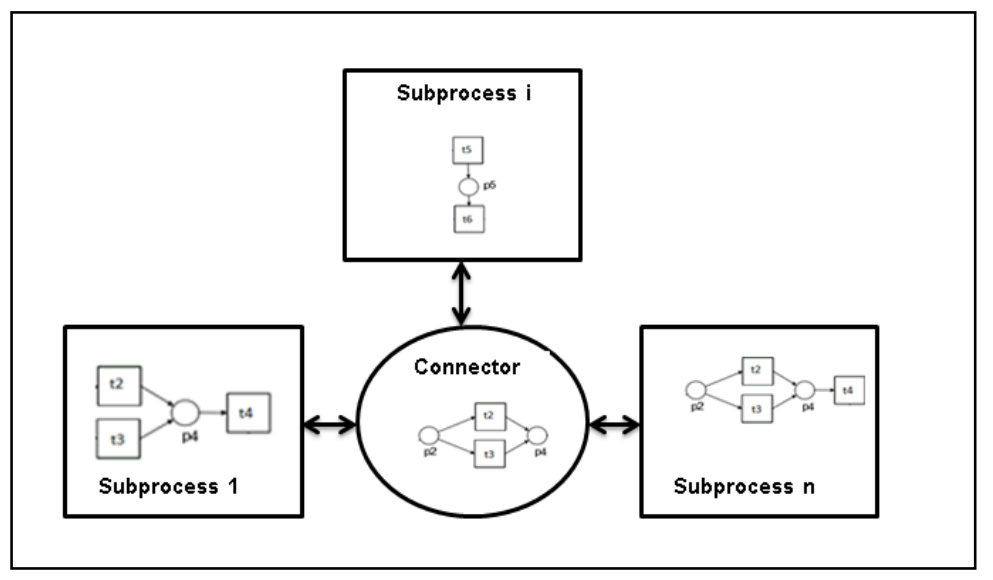

Fig. 4. Communication between separate processes in subprocess - connector.

A main task in subprocess-connector in business process modeling is to produce an abstraction of the process that serves as a basis for detailed definition, study, and possible reengineering to eliminate non-value-added activities. The subprocess-connector must allow for a clear and transparent understanding of the activities being undertaken, the dependencies among the activities, and roles (people, machines, information, etc.) necessary for the process.

Using subprocess-connector, one defines not only the sequence of activities, but also the transmission of data between activities and the conditions that define how the process continues. Since activities might not be executed arbitrarily, they are bound together via connectors.

The execution of subprocess is triggered by start conditions that are determined in the subprocess - connector. The start condition may specify that all incoming control conditions must evaluate to TRUE, or it may specify that at least one of them must evaluate to TRUE. Whatever the start condition is, all incoming conditions must be evaluated before the activity can start. If an activity has no incoming control conditions, it becomes ready when the process or block containing it starts. In addition, a boolean expression called transition condition is associated with each subprocess - connector.

Fig. 5 represents a business process describing the separate stages of fuel oil loading at a filling station. The business process is represented with the help of subprocess - connector. This business process can be described with the following options:

Scenario 1: The driver parks the car, fills oil into the car tank and leaves the filling station.

Scenario 2: In case that the driver is in shortage of money to fill in fuel.

The subprocess - connector is convenient for verifying the target properties of a business process and/or a system as a whole. The complete process provides an opportunity to report a number of deadlock markings, which can prevent serious errors like an incorrectly developed process as part of a series production that can cause dramatic problems to the firm, as well as additional work, legal problems, angry clients, managerial worries and depressed employees. Therefore, it is of crucial importance to check whether a given business process is correct before being started to function.

Deadlock is a state of a system in which no action can take place. Deadlock usually appears in systems that contain subsystems that run in parallel and share some form of common resources. Because Petri nets are a formal model of concurrent systems, they are appropriate for deadlock detection and prevention. Using subprocesses and subprocess - connector allows to simulate the performance of each subprocess and to detect potential deadlock cases in them. This facilitates the deadlock detection in the entire process.

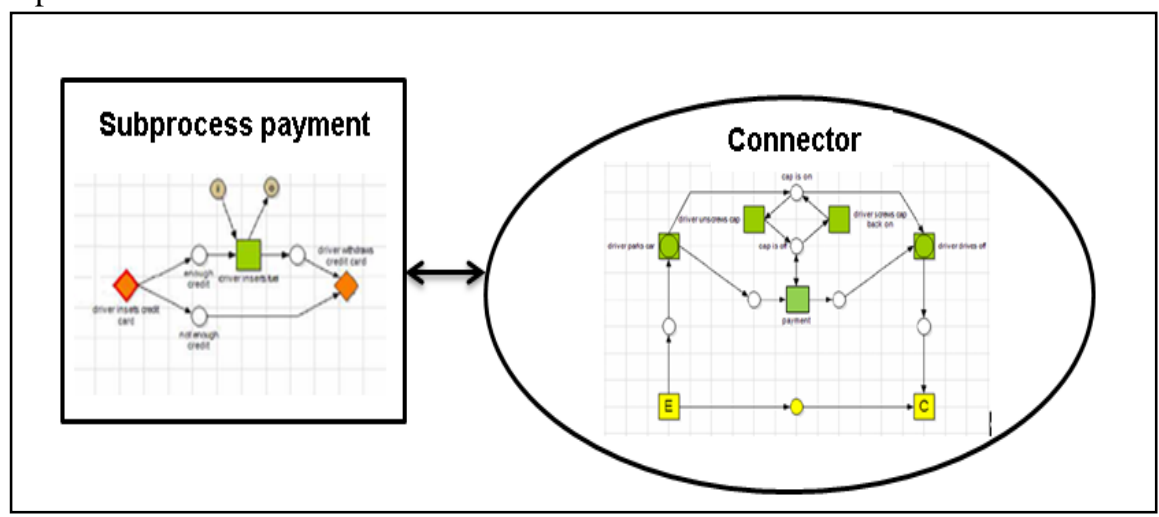

Fig. 5. Communication between separate processes in the subprocess - connector during a concrete business process execution. 
Once the processes describing the whole system have been modeled and each process simulated, they can be united by means of the created subrpocess - connector in order to achieve:

- $\quad$ visualization of processes within the whole system;

- $\quad$ simulation of business processes within the whole system;

- $\quad$ establishing the interaction between separate business processes.

\section{Conclusion}

The paper discusses the use of subprocess-connector that facilitates business process modeling. An approach to detect deadlocks in process models is described. The subprocess-connector requires a deeper understanding of the business process modeling and Petri nets.

Petri nets are a suitable tool for modeling and simulation of business processes within a system. To visualize all business processes in a system a subprocess - connector has been created that helps to detect:

- $\quad$ the deadlock markings in a system;

- the lack of communication between separate subprocesses and the main business process that may cause doubling of a given subprocess;

It is preferable to model initially the entire process of the enterprise and to separate it into several subprocesses and to model them in details. The defined subprocesses also could be divided into their subprocesses if necessary. The entire process leads to optimizing the business processes of the enterprise.

\section{Acknowledgements}

This work is supported by the Bulgarian National Scientific Research Fund under the contract DFNI - I02/13.

\section{References}

[1] Van der Aalst WMP. The Application of Petri Nets to Workflow Management. Department of Mathematics and Computing Science, Eindhoven University of Technology.

[2] Van der Aalst WMP. Putting Petri nets to work in industry. Computers in Industry 1994; 25(1): 45-54

[3] TGI Petri Nets Tools and Software. URL: https://www.informatik.uni-hamburg.de (30.02.2017).

[4] Jensen K. Coloured Petri Nets. Basic concepts, analysis methods and practical use. EATCS monographs on Theoretical Computer Science. Berlin: Springer-Verlag, 1996.

[5] Murata T. Petri nets: Properties, analysis and applications. Proceedings of the IEEE 1989; 77(4): 541-580.

[6] Reisig W. Petri Nets. An Introduction. Berlin: Springer-Verlag, 1999.

[7] Thong WJ, Ameedeen MA. A Survey of Petri Net Tools. Advanced Computer and Communication Engineering Technology. Springer International Publishing, 2015; 537-551.

[8] Vijverberg W. Translation of Process Modeling Languages. Eindhoven, 2006.

[9] Wang J. Petri Nets for Dynamic Event-Driven System Modeling. URL: http://bluehawk.monmouth.edu/ jwang/Ch024.pdf (03.02.2017). 